ASC(Q)

\section{Fazit}

Meine persönliche Bilanz des diesjährigen ASCO-KOngresses:

1. Möglicherweise profitieren doch alle nodal positiven Patientinnen mit einem Vulvakarzinom von einer adjuvanten Bestrahlung.

2. Es bestätigen sich die Daten, dass eine dosisidichte neoadjuvante Chemotherapie bei fortgeschrittenen Zervixkarzinomen hoch effektiv ist. Möglicherweise haben wir bei der medikamentösen Therapie des Zervixkarzinoms einen neuen onkologischen Standard in Form einer Chemotherapie mit Cisplatin und Paclitaxel in Kombination mit Bevacizumab.

3. Der großzügige Einsatz von Metformin, Aspirin und Statinen verbessert insbesondere bei übergewichtigen Patienten die Prognose eines Endometriumkarzinoms.

\section{Prof. Dr. med. Peter Mallmann}

Universitätsklinikum Köln

Klinik und Poliklinik für Frauenheilkunde

und Geburtshilfe

Kerpener Str. 24

50931 Köln

\title{
Fortgeschrittenes Ovarialkarzinom
}

- Nur 20-25\% der Frauen mit einem fortgeschrittenem Ovarialkarzinom überleben ihre Krebserkrankung. Etwa 70\% aller Patientinnen, die zunächst erfolgreich behandelt werden, erleben ein Rezidiv innerhalb eines halben Jahres. Eine aktuelle Phase-III-Studie, die von Prof. Andreas du Bois, Leiter der Abteilung Gynäkologie am Klinikum Essen Mitte, auf dem ASCO präsentiert wurde, sei die erste, in der gezeigt werden konnte, dass mithilfe eines Mulitarget-Tyrosinkinase-Inhibitors die Krankheit kontrolliert werden kann [Du Bois A. et al. J Clin Oncol 2013; 31 (suppl; abstr LBA5503)]. Für die Patientinnen, deren Leben ansonsten sehr schnell wieder vom Krebs bestimmt werde, ein großer Fortschritt, so du Bois, auch wenn ein halbes Jahr gering erscheine.

Für Frauen mit fortgeschrittenem Ovarialkarzinom sind Rezidive an der Tagesordnung. Bislang wurden sie mit einer Chemotherapie in zweiter, dritter oder vierter Linie behandelt. Die Studie zeige, dass Tyrosinkinaseinhibitoren einen substanziellen Einfluss auf das Tumorwachstum haben und den betroffenen Frauen eine signifikant längere krankheitsfreie Zeit schenken, kommentierte Dr. Carol Aghajanian, Leiterin der gynäkologischen Onkologie am Memorial Sloan-Kettering Cancer Center in New York, NY/USA.
In dieser internationalen Studie AGOOVAR16 der Phase III waren 940 Frauen behandelt worden. Die Patientinnen waren an einem fortgeschrittenen epithelialen Tumor des Eierstocks (FiGO-Stadium II-IV ) erkrankt. Sie erhielten über 24 Monate randomisiert als täglich einzunehmende Erhaltungstherapie entweder Pazopanib oder Placebo. Alle Patientinnen waren zuvor R0-operiert worden und hatten sich mehreren Zyklen einer adjuvanten Chemotherapie unterzogen, um das Fortschreiten der Erkrankung zu verzögern. Die Patientinnen wurden durchschnittlich 24 Monate begleitet. Die progressionsfreie Zeit betrug im Pazopanib-Arm median 17,9 Monate, im Placebo-Arm 12,3 Monate. Dies sei ein Zugewinn an krankheitsfreier Zeit von fast einem halben Jahr, erklärte du Bois.

Pazopanib ist ein oraler Inhibitor der Rezeptoren der Tyrosinkinasen VEGF-1, -2, -3, PDGFalpha und beta sowie von c-Kit und greift so in die Signalkaskade ein, welche die Tumorangiogenese steuert, sagte du Bois. In Deutschland ist Pazopanib zur Erstlinientherapie des fortgeschrittenen Nierenzellkarzinoms zugelassen sowie zur Behandlung fortgeschrittener Weichteilsarkome. Du Bois hofft auf eine rasche Zulassung auch beim fortgeschrittenen Ovarialkarzinom, damit möglichst viele Patientinnen von der Erhaltungstherapie profitieren können.

Doris Berger

\section{Weitere Beiträge vom ASCO auf springermedizin.de}

- Weitere Highlights vom diesjährigen

ASCO zu gynäkologischen Tumoren und zu anderen Entitäten sind in unserem Online-Dossier für Sie zusammengefasst unter

www.springermedizin.de/4468404

Dort finden Sie zum Beispiel ausführliche Artikel zu den nachfolgenden MammakarzinomStudien:
BOLERO-2: Patientengruppe mit besonders großem Nutzen

Eine exploratorische Analyse der BOLERO-2-Studie zum Einsatz des oralen mTOR-Inhibitors Everolimus bei HER2negativem, fortgeschrittenem Mammakarzinom ergab, dass Patientinnen mit minimalen genetischen Alterationen in bestimmten Genen besonders von einer Therapie mit Everolimus profitieren.
Therapieoption bei TrastuzumabResistenz

Patientinnen mit HER2-positivem, fortgeschrittenem Mammakarzinom und Trastuzumab-Resistenz haben Vorteile durch eine mTOR-Inhibition. Denn der Nutzen einer Trastuzumab-basierten Therapie kann mittels der mTORInhibition maximiert werden wie aktuelle Daten zeigen. 